
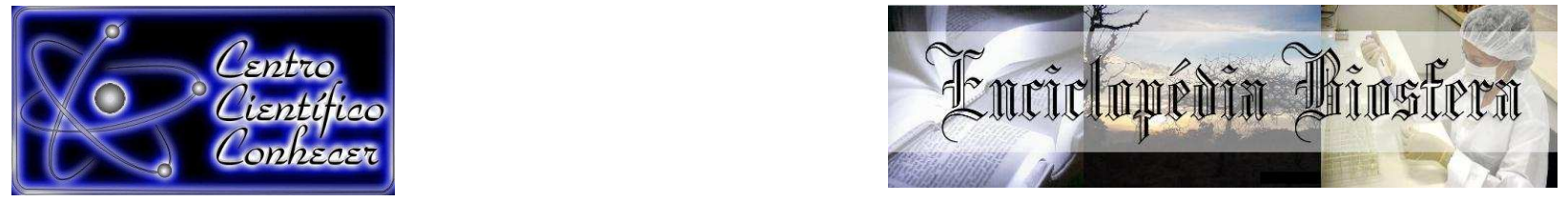

\title{
DISTÚRBIOS DO SONO: NARCOLEPSIA E DISTÚRBIO COMPORTAMENTAL DO SONO REM NOS ANIMAIS
}

Karen Cristina de Oliveira Bastos ${ }^{1}$, Naida Cristina Borges ${ }^{2}$, Adilson Donizeti Damasceno $^{3}$

${ }^{1}$ Mestranda do Programa de Pós-graduação em Ciência Animal, Escola de

Veterinária e Zootecnia da Universidade Federal de Goiás, Goiânia, Brasil kar3nnn@gmail.com

2 Doutora, Professora Associada da Escola de Veterinária e Zootecnia da

Universidade Federal de Goiás, Goiânia, Brasil

3 Doutor, Professor Adjunto da Escola de Veterinária e Zootecnia da Universidade

Federal de Goiás, Goiânia, Brasil

Recebido em: 08/09/2015 - Aprovado em: 14/11/2015 - Publicado em: 01/12/2015

DOI: http://dx.doi.org/10.18677/Enciclopedia_Biosfera_2015_138

\begin{abstract}
RESUMO
A saúde do sono é de extrema importância para o organismo dos animais, pois envolve o desenvolvimento cognitivo, memória, conservação da energia cerebral e processos fisiológicos peculiares. Para preservar essa saúde, é importante obter-se o conhecimento da fisiologia, arquitetura e particularidades desse estado. O sono é o estado de inconsciência reversível, fisiológico, que ocorre de maneira cíclica. É regulado pela interação de processos homeostáticos e ritmo circadiano. Os distúrbios que ocorrem durante o sono promovem a fragmentação, o que causa alterações na arquitetura normal e comprometem o bom funcionamento do organismo. Dentre esses distúrbios, tem-se dois importantes, a narcolepsia e o distúrbio comportamental do sono REM. Os animais foram utilizados como modelo para o estudo desses distúrbios na medicina, porém na ótica da medicina veterinária o conhecimento é limitado. A narcolepsia é caracterizada por episódios de sono intenso, seguidos de fraqueza muscular, alucinações hipnagógicas e paralisia do sono. Sinais esses conhecidos como tétrade clássica. O distúrbio comportamental do sono REM caracteriza-se pela anormal falta de atonia característica da fase REM do sono, o que causa alterações comportamentais, colocando em risco a vida do portador da doença, bem como dos que vivem em sua volta.
\end{abstract}

PALAVRAS-CHAVE: Adenosina, cataplexia, hipocretina, ritmo circadiano.

\section{SLEEP DISORDERS: NARCOLEPSY AND REM BEHAVIOR DISORDER IN ANIMALS - A REVIEW}

\begin{abstract}
A health sleep is extremely important for the body of animals because it involves the cognition, memory, brain energy conservation and peculiar physiological processes. To preserve this health it is important to obtain knowledge of physiology, architecture and particularities of that state. The sleep state is reversible unconsciousness, physiological, which occurs cyclically. It is regulated by the interaction of circadian
\end{abstract}


rhythms and homeostatic processes. The disorders that occur during sleep promote fragmentation, which causes changes in the normal architecture and undermine the proper functioning of the body. Among these disorders has two important, narcolepsy and REM sleep behavior disorder. The animals were used as a model for the study of these disorders in medicine, but in the view of veterinary knowledge is limited. Narcolepsy is characterized by intense episodes of sleep, followed by muscular weakness, paralysis and hypnagogic hallucinations. These signals known as classic tetrad. The REM behavior disorder is characterized by abnormal lack of feature atonia of REM sleep, which causes behavioral changes, endangering the life of the affected animal and those who live around.

KEYWORDS: Adenosine, cataplexy, circadian rhythm, hypocretin.

\section{INTRODUÇÃO}

O sono normal é regulado pela interação de processos homeostáticos com o ritmo circadiano (BROWN et al., 2012). Os processos homeostáticos, que envolvem vias bioquímicas (acúmulo de adenosina e ação da hipocretina), são responsáveis pelo equilíbrio dinâmico do organismo (POSTNOVA et al., 2009) e o ritmo circadiano regido pelo período de claro e escuro (dia e noite) regula o relógio biológico interno por meio do núcleo supraquiasmático localizado no hipotálamo anterior, para influenciar processos fisiológicos (secreção de cortisol e melatonina), metabolismo de biomoléculas (glicose, aminoácidos e ácidos graxos) e o ciclo vigília-sono (JABEEN, 2015).

O ciclo vigília-sono ocorre devido à intensa interação entre neurotransmissores produzidos e liberados no sistema nervoso central (SNC) (KOYAMA, 2012). A vigília ocorre devido à continua ativação do córtex cerebral realizada por neurotransmissores originários do tronco encefálico (LIN et al., 2011). Por conseguinte, a inibição dessa ativação por neurotransmissores inibitórios (ácido gama-aminobutírico e galanina) promove o sono (MOSZCZYNSKI \& MURRAY, 2012).

Sabe-se que o sono é dividido em duas fases distintas. A fase em que não ocorrem movimentos oculares rápidos, denominada NREM (Non-rapid Eyes Movement) e a fase em que ocorrem movimentos oculares rápidos e atonia muscular, o sono REM (Rapid Eyes Movement) (POPAT \& WINSLADE, 2015). A arquitetura normal do sono é sustentada pela variação cíclica dessas duas fases em um período completo de sono (BROWN et al., 2012). A fragmentação desse ciclo pode ocorrer devido a distúrbios específicos do sono (PINTO JR. \& SILVA, 2008). Tal condição gera sono ineficiente, o que afeta a saúde, o temperamento, a cognição e a memória, ou seja, a qualidade de vida dos animais e dos seres humanos (FULLER et al., 2006).

O conhecimento relacionado aos distúrbios do sono e de suas respectivas consequências são recentes, tanto na medicina quanto na medicina veterinária. Contudo, em seres humanos já foram categorizadas cerca de 80 diferentes distúrbios do sono (TOTH \& BHARGAVA, 2013) ao passo que na medicina veterinária de pequenos animais os relatos são raros e incluem a insônia geriátrica, a apnéia obstrutiva do sono, a síndrome da morte súbita, a epilepsia associada ao sono, a narcolepsia e o distúrbio comportamental do sono REM (SCHUBERT et al., 2011). As duas últimas são as mais exploradas na medicina veterinária.

A narcolepsia é um distúrbio neurológico crônico (THORPY \& KRIEGER 2014), que ocorre nos seres humanos e nos animais (LIN et al., 1999) e caracterizase pela ocorrência da tétrade clássica: sonolência excessiva diurna, cataplexia, 
alucinações hipnagógicas e paralisia do sono (AKINTOMIDE \& RICKARDS, 2011). A prevalência da narcolepsia em cães é desconhecida, mas acredita-se que ocorra tal qual em seres humanos. No entanto, esse distúrbio não é bem conhecido pelos profissionais da medicina veterinária ou pelos tutores dos animais. A narcolepsia é uma doença incurável não progressiva, porém com a realização do tratamento adequado, melhora-se a qualidade de vida do animal acometido (TONOKURA et al., 2007).

O distúrbio comportamental do sono REM é uma parassonia que se caracteriza pela presença de movimentos excessivos anormais durante o sono REM (MCCARTER et al., 2012). Esses movimentos podem ser violentos ou bruscos, os quais podem apresentar riscos aos próprios animais acometidos e às pessoas ou outros animais próximos (TROTTI, 2010). Há poucos relatos de casos do distúrbio em cães e gatos (SCHUBERT et al., 2011) e o tratamento ainda não é consolidado na medicina (TROTTI, 2010), tampouco na medicina veterinária. Há necessidade de pesquisas relacionadas a esse distúrbio, uma vez que não se conhece a prevalência, predisposição racial e tratamento adequado.

Os distúrbios do sono, no ponto de vista da medicina veterinária, são obscuros e pouco explorados. Por isso, o profissional ao deparar-se com pacientes que apresentem alterações características desses distúrbios, pode ter dificuldades na detecção e no estabelecimento do tratamento adequado devido à falta de conhecimento. Neste sentido, propõe-se neste trabalho a apresentação de elementos que descrevam os padrões normais do sono em animais, bem como os aspectos clínicos da narcolepsia e do distúrbio comportamental do sono REM.

\section{REVISÃO DE LITERATURA}

\section{SONO NORMAL}

O sono é um comportamento fisiológico cíclico notadamente reconhecido pela redução reversível do nível de consciência (MAQUET, 2001). Trata-se do resultado de processos cerebrais ativos que ocorrem nos animais devido ao circuito neural organizado localizado no sistema nervoso central (MARKOV \& GOLDMAN, 2006). Sabe-se que dormir é importante para a restauração de energia cerebral (KRUEGER et al., 2015), consolidação de memória, desenvolvimento da capacidade cognitiva (TONONI \& CIRELLI, 2014), desintoxicação do cérebro (INOUE et al., 1995), restauração celular, bom funcionamento do sistema endócrino, equilíbrio do sistema nervoso central e síntese proteica (LAILA \& BAHAMMAM, 2011). A interação entre processos homeostáticos e ritmo circadiano regula o ciclo vigília-sono (PRIMHAK \& KINGSHOTT, 2012).

O ritmo circadiano é o regulador dos sistemas fisiológico e endócrino do organismo dos animais. O relógio biológico central localizado no núcleo supraquiasmático do hipotálamo anterior comanda o relógio biológico periférico presentes nos tecidos dos órgãos, o que rege comportamento, metabolismo e processos fisiológicos. Os períodos de claro e escuro são determinantes para a secreção do hormônio melatonina, o principal sincronizador endógeno e indutor do sono. A incidência luminosa inibe a produção e a liberação da melatonina pela glândula pineal, o que garante a sua atuação exclusivamente no período noturno em todas as espécies do reino animal (REITER et al., 2011).

Os processos homeostáticos estão relacionados a substâncias que são produzidas no organismo, as quais são capazes de induzir e manter o sono ou a vigília (PRIMHAK \& KINGSHOTT, 2012). A substância denominada adenosina é o 
subproduto da adenosina-trifosfato (ATP). Essa substância acumula-se no prosencéfalo basal ao longo de um período prolongado de vigília, devido à grande demanda energética do SNC. Esse acúmulo inibe áreas cerebrais responsáveis pela manutenção da vigília, o que, consequentemente, induz o sono (BJORNESS \& GREENE, 2009). Outra substância que participa dos processos homeostáticos é o neuropeptídio denominado hipocretina produzido por células neurais hipocretinérgicas localizadas no hipotálamo lateral (CHEN et al., 2014). Também conhecida por orexina, a hipocretina desempenha papel importante na indução e manutenção da vigília (BLASIAK et al., 2015). As células nervosas responsáveis pela produção e liberação das hipocretinas estão presentes no principal sistema neural responsável pela vigília, o sistema reticular ativador ascendente (SRAA). Com isso, o papel dessa substância é de promover a excitação das células nervosas presentes no SRAA, a fim de otimizar a ação dessas células, para que ocorra o despertar e mantenha-se a vigília (SAPER et al., 2001). Portanto, a ação das hipocretinas durante o sono é nula (ESPANA \& SCAMMELL, 2011).

O SRAA é responsável por gerar e manter a vigília e está localizado no tronco encefálico (Figura 1). Núcleos e projeções da formação reticular compõem o SRAA (JANG \& KWON, 2015). Esses núcleos são divididos em colinérgicos e monoaminérgicos. Os núcleos colinérgicos, compostos pelas áreas tegmentares laterodorsal e peduculopontino, são responsáveis pela produção da acetilcolina. $O$ prosencéfalo basal (PB) também possui células neurais colinérgicas. Os componentes monoaminérgicos são: lócus cerúleos, núcleos da rafe, neurônios da região periaquedutal ventral da substância cinzenta, núcleo tuberomamilar do hipotálamo e hipotálamo lateral. Esses núcleos são responsáveis pela produção da noradrenalina, serotonina, dopamina, histamina e hipocretina, respectivamente (BROWN et al., 2012). A vigília que ocorre nos animais se deve a ativação do córtex cerebral devido à atividade desses neurotransmissores no circuito tálamo-cortical (YEO et al., 2013).

O sono ocorre devido à inibição exercida pelo ácido gama-aminobutírico (GABA) e pela galanina no SRAA (Figura 1). Esses inibidores são produzidos no núcleo pré-óptico ventrolateral (VLPO) (MOSZCZYNSKI \& MURRAY, 2012). A adenosina estimula a atividade inibitória do VLPO (PORKKA-HEISKANEN et al., 1997).

O sono divide-se em duas fases distintas: REM e NREM (POPAT \& WINSLADE, 2015). O sono NREM é a fase mais duradoura do sono e é considerada a etapa restauradora e profunda, em que há atividade elétrica cerebral de baixa frequência e alta amplitude, as chamadas ondas lentas do sono (SANTOS et al., 2014). Nessa fase, ocorre redução do consumo de energia, do metabolismo do sistema nervoso central, da atividade do sistema nervoso autônomo e do tônus muscular (HASAN \& ALÓE, 2011). O sono REM é a fase em que ocorrem os sonhos. Nessa etapa há atonia muscular, frequências cardíaca e respiratória irregulares, pressão arterial oscilante (SANTOS et al., 2014), ativação do sistema autônomo e aumento do metabolismo cerebral. O sono REM associa-se à capacidade de aprendizado, consolidação da memória e síntese de proteínas (HASAN \& ALÓE, 2011).

O sono normal consiste na variação cíclica dessas duas fases (HASAN \& ALÓE, 2011). O sono inicia-se com o sono NREM. O VLPO realiza a inibição de todos os componentes do SRAA, o que diminui progressivamente a atividade serotoninérgica, noradrenérgica, dopaminérgica, histaminérgica e anula a atividade hipocretinérgica durante o sono NREM (STEININGER et al., 2001). Sabe-se que a 
serotonina e noradrenalina cumprem o papel de inibir a atividade colinérgica promotora de REM (BROWN et al., 2012). Porém, quando o VLPO inibe a atividade dos neurônios serotoninérgicos e noradrenérgicos, ocorre a desinibição do sistema colinérgico promotor do sono REM, o que leva a ocorrência do sono REM (SAPER et al., 2001).

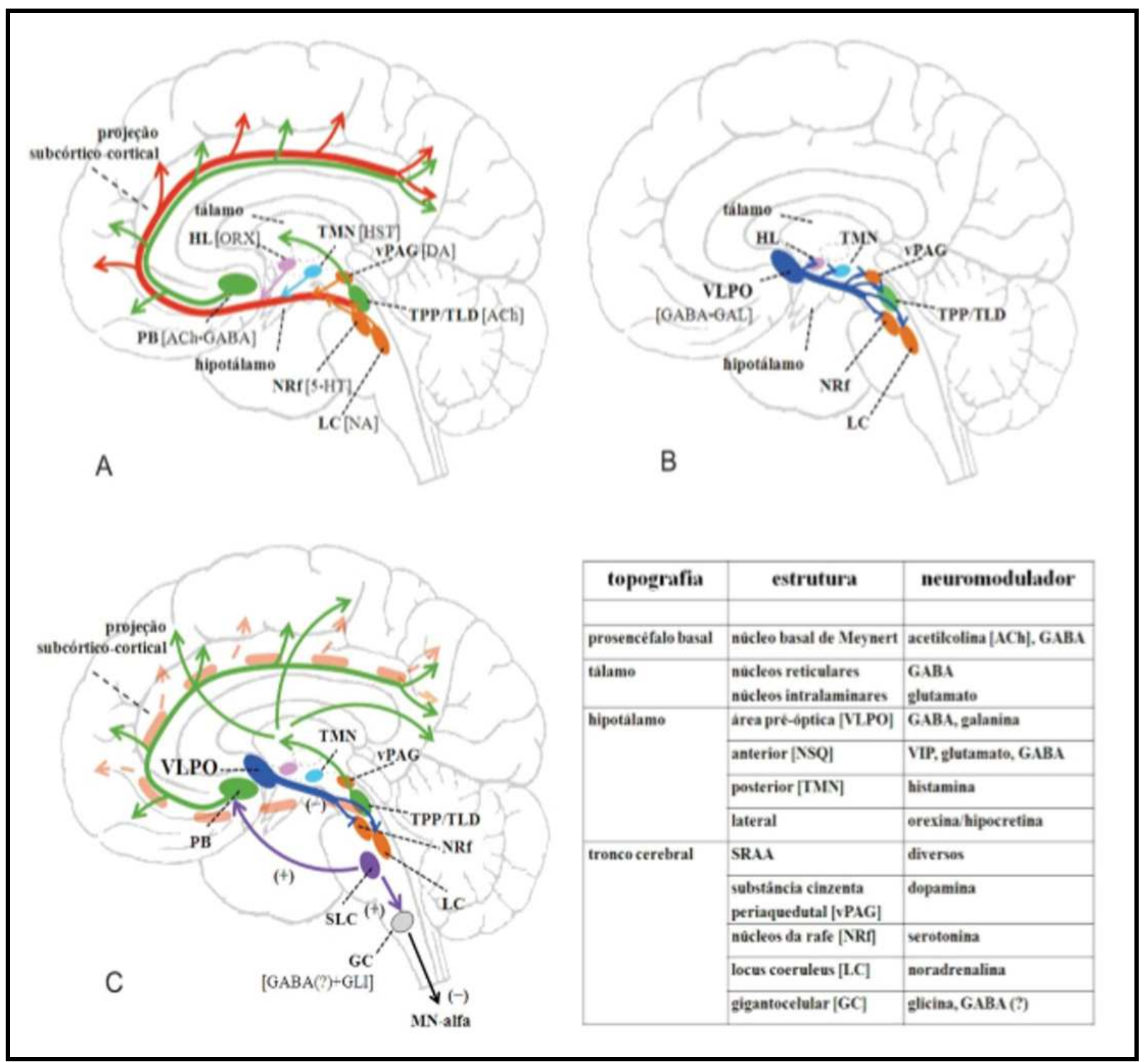

FIGURA 1 Esquema representativo do mecanismo de controle neurais do ciclo vigília-sono. A) Promoção da vigília pela ativação cortical pelos componentes do SRAA, por meio do circuito tálamo-cortical demonstrado pela cor vermelha. Em alaranjado, o lócus cerúleos (LC) e o núcleos da Rafe (NRf) e região periaquedutal ventral (vPAG). Em lilás, o hipotálamo lateral (HL). Em azul, o núcleo tuberomamilar do hipotálamo (TMN). Em verde, os núcleos tegmentares peduculopontino e laterodorsal (TPP e TLD, respectivamente) e o prosencéfalo basal. B) Promoção do sono NREM. Em azul escuro, o núcleo pré-óptico ventrolateral (VLPO) inibindo áreas promotoras da vigília (TMN, TPP, TLD, NRf, LC e vPAG). C) Promoção do sono REM. O VLPO inibindo o NRf e LC. Região da ponte encefálica (SLO sublocus cerúleos) estimulando o PB e os núcleos TPP e TLD na ativação cortical característica do sono REM e estimulando o GABA na atonia muscular nos neurônios motores alfa. Quadro) Demonstrativo das estruturas e os respectivos neurotransmissores participantes do ciclo de vigília-sono.

Fonte: Adaptado de GOMES et al., (2010). 


\section{DISTÚRBIOS DO SONO \\ NARCOLEPSIA}

A palavra narcolepsia provém do grego narke que significa estupor e lepsis que significa crise e define uma doença neurológica crônica caracterizada pela instabilidade dos estados de vigília-sono e pela intrusão anormal do sono REM na vigília (KUMAR \& SAGILI, 2014), que resulta em sonolência excessiva diurna, cataplexia, paralisia do sono e alucinações hipnagógicas, denominada tétrade clássica da narcolepsia (TOTH \& BHARGAVA, 2013).

O termo foi empregado primeiramente pelo físico francês Gélineau, no final do século XIX. Até a primeira metade do século $X X$, não haviam pesquisas relacionadas à narcolepsia. Em 1934, Yoss e Daly observaram a ocorrência dos sinais associados à narcolepsia. Na década de 50 , o sono REM foi descoberto por Kleitman, contudo, a associação entre o sono REM e a narcolepsia foi possível graças aos estudos de Vogel (KUMAR \& SAGILI, 2014). Em estudo realizado em cães por LIN et al. (1999), associou-se a hipocretina à fisiopatogenia da narcolepsia.

A narcolepsia ocorre nos seres humanos e em outras espécies de mamíferos, como cães, gatos, ratos (SAKURAI, 2013), equinos, ovelhas e bovinos (CHEN et al., 2009). Caracteriza-se clinicamente pela ocorrência de episódios súbitos de fraqueza muscular (cataplexia), hipersonolência, paralisia do sono (incapacidade de realizar movimentos voluntários no momento em que se desperta após episódio breve de sono) e alucinações hipnagógicas que consistem de percepções visuais ou auditivas anormais quando ocorre a intrusão do sono ou o despertar (ST LOUIS, 2014).

Os sinais clínicos da narcolepsia são semelhantes em cães e seres humanos. Existem duas formas diferentes de apresentação da doença: esporádica e familial. A forma esporádica, tanto em seres humanos, quanto em cães, é a forma que mais se expressa (HUNGS et al., 2001). Essa forma, também conhecida por adquirida, ocorre devido à perda da capacidade de produção da hipocretina por células neurais hipocretinérgicas, por causas ainda desconhecidas. A análise do líquor permite a detecção da ausência ou diminuição desse neuropeptídio, o que corrobora o diagnóstico de narcolepsia (RIPLEY et al., 2001). Em contrapartida, a forma familial ocorre devido ao padrão genético autossômico recessivo em que ocorre a mutação nos receptores de hipocretina (LIN et al., 1999). No cão, essa forma ocorre aproximadamente no sexto mês de idade, mas pode ser expressa precocemente, antes dos quatro meses (TONOKURA et al., 2007).

Os cães acometidos pela doença são pouco ativos, sonolentos e apresentam latência diminuída para o início do sono. Os ataques de cataplexia são os sinais mais comuns da narcolepsia nos cães e ocorrem quando há estímulos emotivos, como a oferta de alimentos palatáveis, a alimentação da rotina ou brincadeiras que levam à euforia. Esses ataques começam com a fraqueza dos membros pélvicos e, logo após, ocorre flacidez do pescoço, o que ocasiona a queda do animal ao chão. Comumente, os cães permanecem de olhos abertos e conscientes durante 0 episódio, sobretudo no começo (Figura 2). Esses episódios podem durar de segundos a minutos e, geralmente, logo após a ocorrência, os cães tentam levantar e andar, mas apresentam incoordenação e podem cair novamente. Pode-se interromper os ataques por meio de estímulos auditivos e contato, como chamar o animal pelo nome e afagá-lo. Quando o tempo do episódio é longo, o animal adormece e há a intrusão do sono REM. Outros sinais da tétrade clássica, como a paralisia do sono e as alucinações hipnagógicas, podem ocorrer nos cães, contudo não há maneiras objetivas de identificá-las (TONOKURA et al., 2007). 


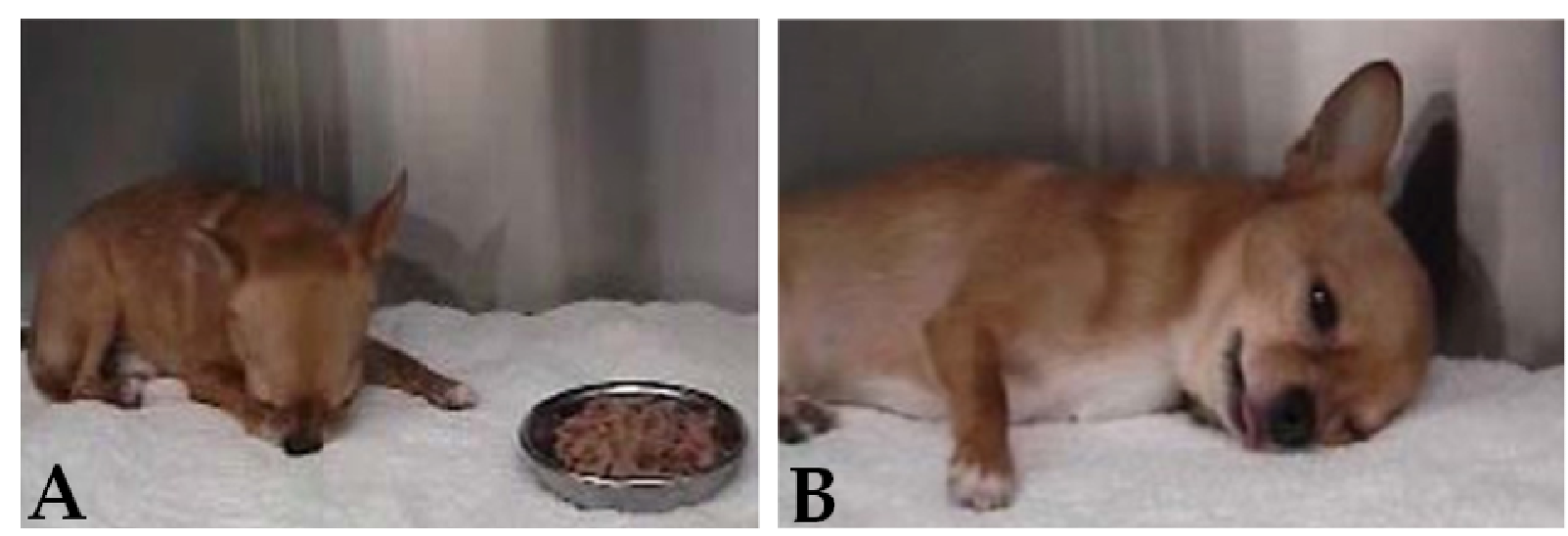

FIGURA 2 Cão da raça Chihuahua com cataplexia. A) Diante do alimento houve a ocorrência da crise de cataplexia em que a cabeça encontra-se caída em virtude da flacidez do pescoço além da perda generalizada do tônus muscular. B) A observação dos olhos abertos permite inferir que o animal encontra-se, aparentemente, consciente durante ataque cataplético.

Fonte: Adaptado de TONOKURA et. al., (2007)

Análises realizadas por KUSHIDA et al., (1985), em registros feitos a partir de polígrafo utilizado em cães com narcolepsia, revelaram três diferentes estágios do ataque cataplético. O primeiro estágio foi caracterizado por atonia muscular, ondas cerebrais características da vigília e movimentos oculares (acompanhamento visual de movimentos). No segundo estágio, foram detectadas ondas cerebrais teta (de frequência entre 4-7 Hz) características do sono REM. No terceiro estágio, detectaram-se ondas cerebrais que caracterizavam a transição do sono para a vigília. KAITIN et al., (1986) realizaram análises a partir de registros de polígrafo em cães narcolépticos e constataram que o sono desses cães é fragmentado.

Sabe-se que, comumente, durante o sono REM ocorre atonia muscular. Essa atonia se dá pela inibição gabaérgica e glicinérgica nos neurônios motores da medula espinhal. Sinais glutamatérgicos provenientes de células neurais localizadas na região sublaterodorsal excitam essas células inibitórias gabaérgicas e glicinérgicas. Durante a vigília, as células glutamatérgicas são inibidas por neurônios gabaérgicos das regiões periaquedutal ventrolateral da substância cinzenta e tegmento pontino lateral. Porém, na cataplexia, os neurônios gabaérgicos dessas regiões são inibidos. Com isso, as células glutamatérgicas são desinibidas, o que causa excitação das células gabaérgicas e glicinérgicas que inibem os neurônios motores, o que leva a atonia da narcolepsia. As emoções desencadeiam a cataplexia devido à presença de projeções límbicas excitatórias na região sublaterodorsal (células glutamatérgicas) e projeções límbicas inibitórias nas regiões periaquedutal ventrolateral e tegmento pontino lateral. Além disso, sabe-se que neurônios hipocretinérgicos projetam-se para os neurônios motores e os excitam. $\mathrm{Na}$ narcolepsia, com a deficiência de hipocretina ou ineficiência dos receptores de hipocretina, essa excitação é ineficiente, o que leva a diminuição do tônus muscular (KUMAR \& SAGILI, 2014).

O diagnóstico da narcolepsia em cães é baseado, habitualmente, na detecção da cataplexia, devido a grande incidência desse sinal clínico em animais acometidos, além da facilidade de identificação da ocorrência do mesmo. Na rotina clínica, pode-se aplicar o teste chamado food-elicited cataplexy test (FECT), que consiste em dispor 12 pedaços de comida canina alinhados no chão para mensuração do tempo de preensão e deglutição. No cão normal, espera-se que 
ocorra a ingestão de todos os pedaços em aproximadamente dez segundos. Por sua vez, o cão narcoléptico se excita ao contato com a comida, desencadeando a cataplexia. Esse teste, além de auxiliar no diagnóstico da narcolepsia, também é utilizado para testar a eficácia do tratamento prescrito em cada paciente e realizar o ajuste da dose, caso necessário (NISHINO et al., 1994).

A anamnese e o histórico clínico são de suma importância no auxílio do diagnóstico da doença. O tutor pode auxiliar na detecção da narcolepsia ao fornecer informações detalhadas sobre quando e como ocorrem os sinais clínicos, cabendo ao médico veterinário determinar se os comportamentos são característicos da doença (NISHINO \& MIGNOT, 1997).

Ao estabelecer o diagnóstico conclusivo da narcolepsia, é importante informar ao tutor de que se trata de uma doença que não tem cura, porém não é progressiva, não estabelece risco à vida e que se tratada corretamente, melhorará a qualidade de vida do paciente. Para tanto, o sinal clínico que requer maior atenção é a cataplexia (TONOKURA et al., 2007).

A ativação do sistema adrenérgico é eficaz no controle da cataplexia. Para tanto, os antidepressivos tricíclicos, como a imipramina, na dose de 1,5-3,0 mg/kg/dia e a clomipramine, na dose de 3,0-6,0 mg/kg/dia, vem sendo empregados. Outro medicamento que possui efeito anticataplético é a ioimbina, antagonista seletivo dos receptores alfa-2 adrenérgicos, na dose $0,15-0,30 \mathrm{mg} / \mathrm{kg}$, duas vezes ao dia. $\mathrm{A}$ ioimbina ativa a transmissão adrenérgica pré-sináptica, tem ação estimulante em cães e roedores e se mostrou eficaz no tratamento a longo prazo da sonolência excessiva e cataplexia que ocorrem na narcolepsia. A escolha do fármaco e o ajuste da dose são realizados com base na observação da frequência e do tempo utilizado pelo animal para terminar uma refeição (NISHINO \& MIGNOT, 1997).

\section{DISTÚRBIO COMPORTAMENTAL DO SONO REM}

Durante o sono REM, as células neurais inibitórias localizadas na ponte encefálica e medula oblonga, exercem forte inibição da atividade dos neurônios motores inferiores no tronco cerebral e cordão espinhal, com a finalidade de prevenir movimentos excessivos que podem ocorrer nessa fase do sono (FRAIGNE et al., 2015). O distúrbio comportamental do sono REM (DCR) caracteriza-se pela falta dessa inibição, o que ocasiona alta ativação anormal do córtex cerebral. Essa atividade cortical estimula os neurônios motores inferiores, o que provoca movimentos violentos dos membros, agressividade e mudanças comportamentais (BOEVE et al., 2007). Acredita-se que essa atividade motora atípica durante o sono REM está associada com manifestações relacionadas aos sonhos, ou seja, capacidade de praticar movimentos similares aos que ocorrem durante o sonho (LUPPI et al., 2013).

A descoberta dos movimentos rápidos dos olhos característicos do sono REM, foi publicada por Kleitman em 1953 (AKERSTEDT \& NILSSON, 2003). HENLEY \& MORRISON (1974), realizaram a transecção da junção pontomesencefálica em gatos e observaram que o sono REM e a atonia muscular característica dessa fase cessaram. Nesse trabalho, também foi realizada a transecção da junção pontomedular e notou-se que não houve alteração na ocorrência normal do sono REM, bem como da atonia muscular característica. Com isso, concluiu-se que os geradores da atonia muscular do sono REM localizavam-se na ponte encefálica. 
No experimento realizado por BOISSARD et al., (2002), aplicou-se um agonista do glutamato no núcleo sublaterodorsal em ratos, o que induziu a atonia característica do sono REM, demonstrando o importante papel do glutamato nesse evento. Portanto, sabe-se que células glutamatérgicas são responsáveis por excitarem células neurais medulares, que contém GABA, causando inibição dos neurônios motores inferiores (KUMAR \& SAGILI, 2014). Além disso, LU et al., (2006) mostraram que lesões citotóxicas no núcleo sublaterodorsal e regiões adjacentes resultaram em sono REM sem atonia muscular, o que ressalta o importante papel dessa região na atonia característica do sono REM.

O entendimento da fisiopatogenia do DCR foi possível com a realização dos trabalhos anteriormente relatados. Concluiu-se que o DCR ocorre, nos seres humanos e nos animais, devido às lesões ou às mutações genéticas que afetam o núcleo sublaterodorsal localizado no tronco encefálico. Sabe-se que as células neurais glutamatérgicas estão presentes nessa região. Portanto, danos no núcleo laterodorsal comprometem a atividade dessas células na promoção da atonia muscular característica do sono REM (KRENZER et al., 2013).

As manifestações clínicas do DCR podem ser agudas ou crônicas (SCHENCK \& MAHOWALD, 2002). A forma aguda ocorre de maneira transitória e são observadas após a ingestão de determinadas drogas e, particularmente, nos seres humanos, com a abstinência do álcool. A manifestação crônica pode ser de origem idiopática ou secundária (BOEVE et al., 2007). O paciente com DCR idiopático não apresenta doença neurológica prévia (GAGNON et al., 2009). As causas secundárias do DCR são: degenerativas, congênitas, neoplásicas, infecciosas, traumáticas ou de origem vascular (PAULETTO et al., 2004).

Em seres humanos, os sinais clínicos característicos do DCR envolvem alterações comportamentais durante o sono REM. A pessoa acometida pode cantar, falar, chutar, agarrar pessoas ou objetos, estrangular e pular da cama. Podem ser observados comportamentos violentos, o que coloca em risco a vida tanto da pessoa acometida quanto de pessoas que dormem próximas a elas (MCGRANE et al., 2015).

Em cães, os sinais clínicos foram relatados em trabalho realizado por SCHUBERT et al., (2011). Quatorze cães acometidos pela doença apresentaram movimentos violentos dos membros, em decúbito lateral ou esternal, mastigação, ranger de dentes, tentativa de morder o ar ou objetos próximos, ataques ao tutor ou animais próximos.

Segundo a Classificação Internacional de Distúrbios do Sono, o diagnóstico do DCR nos seres humanos é baseado no histórico clínico e no exame de polissonografia. A polissonografia é o registro simultâneo de eventos fisiológicos durante 0 sono. Analisa-se atividade elétrica cerebral, muscular, cardíaca, respiratória, dentre outras (PINTO JR. \& SILVA, 2008). O achado na polissonografia característico do DCR é a presença de tônus muscular elevado durante atividade elétrica cerebral característica do sono REM sem atividade epileptiforme no traçado do eletroencefalograma (EEG) (SCHENCK et al., 1993). Na medicina veterinária, a polissonografia ainda não é uma realidade. Com isso, o diagnóstico é realizado baseando-se no relato do tutor de alterações no comportamento e vídeomonitorização do sono do animal (SCHUBERT et al., 2011).

O tratamento para o DCR ainda não é consolidado na medicina, tampouco na medicina veterinária. Em seres humanos, o clonazepam (benzodiazepínico) é o tratamento mais utilizado. Porém, a melatonina é considerada outra opção de tratamento, embora não haja estudos que estabeleça um paralelo entre ambos. 
Acredita-se que a melatonina restaura a atonia muscular no sono REM, mas o mecanismo de ação ainda é desconhecido (MCGRANE et al., 2015) .

No trabalho de SCHUBERT et al., (2011), há relatos de que a melatonina associada ao fenobarbital reduziu levemente os sinais do DCR, mas não padronizou-se dosagens dos medicamentos. Realizou-se a administração do brometo de potássio, na dose de $44 \mathrm{mg} / \mathrm{kg} / \mathrm{dia}$ em $64 \%$ dos cães acometidos pelo DCR. Relatou-se que houve a diminuição da intensidade e a frequência dos eventos. No entanto, notou-se sedação como efeito colateral em dois cães que usaram o brometo de potássio. Nesses casos, associou-se o brometo de potássio à melatonina e em somente um dos casos verificou-se a melhora da sedação. $O$ clonezepam, utilizado em seres humanos, não se mostrou eficaz. O mecanismo de ação dos medicamentos ainda não é conhecido. Necessita-se de mais pesquisas para o controle adequado desse distúrbio em cães.

Outras observações importantes demonstradas por SCHUBERT et al., (2011), estão relacionadas ao sexo, idade e raças. Em paralelo aos seres humanos, em que o distúrbio ocorre majoritariamente em homens, notou-se nesse trabalho que $57 \%$ dos animais acometidos eram fêmeas, além do autor citar outro trabalho em que se detectou o DCR em fêmea. Dos cães estudados durante os nove anos de realização do trabalho, 29\% deles eram da raça Golden retriever. Outras raças categorizadas foram: SRD, Dálmata, Rotweiller, Schnauzer, Bichon frise, Blue heeler, Queensland heeler, Pastor alemão e Italian sinone. Por fim, outro paralelo estabelecido entre a ocorrência do DCR em seres humanos e cães é de que nos seres humanos o DCR ocorre, geralmente, depois dos 50 anos e nos cães, a maior ocorrência é em animais jovens (menos de dois anos).

\section{CONSIDERAÇÕES FINAIS}

A saúde do sono é imprescindível para o equilíbrio dinâmico do organismo, pois permite que se desenvolva, adequadamente, memória, capacidade cognitiva, balanço hormonal e constituição corporal. Por isso, o estudo dos distúrbios do sono dos animais é de extrema importância e os fatores que fragmentam e prejudicam o sono devem ser devidamente identificados.

Os cães foram utilizados nas pesquisas relacionadas ao sono ao longo dos anos e foram modelos de estudo a fim de mimetizar a narcolepsia, dentre outros distúrbios na medicina. Porém, a ótica médica veterinária em relação aos distúrbios do sono no cão é escassa, com isso, esses distúrbios têm grande impacto na qualidade de vida do animal acometido não diagnosticado e não tratado.

Os relatos de casos associados aos distúrbios do sono em cães são raros. A baixa prevalência associada ao desconhecimento do profissional em diagnosticar as doenças do sono é o provável motivo para que a medicina veterinária encontre-se em patamar abaixo em relação à medicina. Em face disso, vislumbra-se a necessidade de inserir a disciplina de fisiologia e distúrbios do sono na graduação e estimular mais pesquisas na área por médicos veterinários, principalmente, na adequação dos meios de diagnóstico já existentes para humanos na rotina clínica veterinária e no estabelecimento de protocolos terapêuticos eficientes para o seu controle.

\section{REFERÊNCIAS}

AKERSTEDT, T.; NILSSON, P. M. Sleep as restitution: an introduction. Journal of Internal Medicine, v. 254, p. 6-12, 2003. 
AKINTOMIDE, G. S.; RICKARDS, H. Narcolepsy: a review. Neuropsychiatr Dis Treat, v. 7, p. 507-18, 2011.

BLASIAK, A.; SIWIEC, M.; GRABOWIECKA, A.; BLASIAK, T.; CZERW, A.; BLASIAK, E.; KANIA, A.; RAJFUR, Z.; LEWANDOWSKI M. H.; GUNDLACH A. L. Excitatory orexinergic innervation of rat nucleus incertus - implications for ascending, motivation arousal and feeding control. Neuropharmacology, Aug 82015.

BOEVE, B. F.; SILBER, M. H.; SAPER, C. B.; FERMAN, T. J.; DICKSON, D. W.; PARISI, J. E.; BENARROCH, E. E.; AHLSKOG, J. E.; SMITH, G. E.; CASELLI, R. C.; TIPPMAN-PEIKERT, M.; OLSON, E. J.; LIN, S. C.; YOUNG, T.; WSZOLEK, Z.; SCHENCK, C. H.; MAHOWALD, M. W.; CASTILLO, P. R.; DEL TREDICI, K.; BRAAK, H. Pathophysiology of REM sleep behaviour disorder and relevance to neurodegenerative disease. Brain, v. 130, n. Pt 11, p. 2770-88, Nov 2007.

BOISSARD, R.; GERVASONI, D.; SCHMIDT, M. H., BARBAGLI, B., FORT, P.; LUPPI, P. H. The rat ponto-medullary network responsible for paradoxical sleep onset and maintenance: a combined microinjection and functional neuroanatomical study. Eur J Neurosci, v. 16, n. 10, p. 1959-73, Nov 2002.

BROWN, R. E.; BASHEER, R.; MCKEENA, J. T.; STRECKER, R. E.; MCCARLEY, R. W. Control of sleep and wakefulness. Physiol Rev, v. 92, n. 3, p. 1087-187, Jul 2012.

CHEN, L.; BROWN, R. E.; MCKEENA, J. T.; MCCARLEY, R. W. Animal models of narcolepsy. CNS and Neurological Disorders - Drug Targets, v. 8, n. 4, p. 296308, 2009.

CHEN, Q.; DE LECEA, L.; HU, Z.; GAO, D. The Hypocretin/Orexin System: An Increasingly Important Role in Neuropsychiatry. Med Res Rev, Jul 122014.

ESPANA, R. A.; SCAMMELL, T. E. Sleep neurobiology from a clinical perspective. Sleep, v. 34, n. 7, p. 845-58, Jul 2011.

FRAIGNE, J. J.; TORONTALI, Z. A.; SNOW, M. B.; PEEVER, J. H. REM Sleep at its Core - Circuits, Neurotransmitters, and Pathophysiology. Front Neurol, v. 6, p. 123, 2015.

FULLER, P. M.; GOOLEY, J. J.; SAPER, C. B. Neurobiology of the sleep-wake cycle: sleep architecture, circadian regulation, and regulatory feedback. J Biol Rhythms, v. 21, n. 6, p. 482-93, Dec 2006.

GAGNON, J. F.; VENDETTE, M.; POSTUMA, R. B.; DESJARDINS, C.; MASSICOTTE-MARQUEZ, J.; PANISSET, M.; MONTPLAISIR, J. Mild cognitive impairment in rapid eye movement sleep behavior disorder and Parkinson's disease. Ann Neurol, v. 66, n. 1, p. 39-47, Jul 2009.

GOMES, M. M.; QUINBONES, M. S.; ENGELHARDT, E. Neurofisiologia do sono e aspectos farmacoterapêuticos dos seus transtornos. Rev Bras Neurol, v. 46, n. 1, p. 5-15, 2010.

HASAN, R.; ALÓE, F. Updates on the sleep-wake cycle. Sleep Sci, v. 4, n. 2, p. 5260, 2011. 
HENLEY, K.; MORRISON, A. R. A re-evaluation of the effects of lesions of the pontine tegmentum and locus coeruleus on phenomena of paradoxical sleep in the cat. Acta Neurobiol Exp (Wars), v. 34, n. 2, p. 215-32, 1974.

HUNGS, M.; FAN, J.; LIN, L.; LIN, X.; MAKI, R. A.; MIGNOT, E. Identification and functional analysis of mutations in the hypocretin (orexin) genes of narcoleptic canines. Genome Res, v. 11, n. 4, p. 531-9, Apr 2001.

INOUE, S.; HONDA, K.; KOMODA, Y. Sleep as neuronal detoxification and restitution. Behav Brain Res, v. 69, n. 1-2, p. 91-6, Jul-Aug 1995.

JABEEN, S. Effect of Sleep/Wake Cycle on Autonomic Regulation. J Coll Physicians Surg Pak, v. 25, n. 6, p. 434-7, Jun 2015.

JANG, S. H.; KWON, H. G. The ascending reticular activating system from pontine reticular formation to the hypothalamus in the human brain: a diffusion tensor imaging study. Neurosci Lett, v. 590, p. 58-61, Mar 172015.

KAITIN, K. I.; KILDUFF, T. S.; DEMENT, W. C. Sleep fragmentation in canine narcolepsy. Sleep, v. 9, n. 1 Pt 2, p. 116-9, 1986.

KOYAMA, Y. [Regulation of sleep and wakefulness through the monoaminergic and cholinergic systems]. Brain Nerve, v. 64, n. 6, p. 601-10, Jun 2012.

KRENZER, M.; LU, J.; MAYER, G.; OERTEL, W. From bench to bed: putative animal models of REM sleep behavior disorder (RBD). J Neural Transm, v. 120, n. 4, p. 683-8, Apr 2013.

KRUEGER, J. M.; FRANK, M.; WISOR, J.; ROY, S. Sleep Function: Toward Elucidating an Enigma. Sleep Medicine Reviews, 2015.

KUMAR, S.; SAGILI, H. Etiopathogenesis and neurobiology of narcolepsy: a review. J Clin Diagn Res, v. 8, n. 2, p. 190-5, Feb 2014.

KUSHIDA, C. A.; BAKER, T. L.; DEMENT, W. C. Electroencephalographic correlates of cataplectic attacks in narcoleptic canines. Electroencephalogr Clin Neurophysiol, v. 61, n. 1, p. 61-70, Jul 1985.

LAILA, A.; BAHAMMAM, A. S. Metabolic, Endocrine, and Immune Consequences of Sleep Deprivation. Open Respir Med, v. 5, p. 31-43, 2011.

LIN, J. S.; ANACLET, C.; SERGEEVA, O. A.; HAAS, H. L. The waking brain: an update. Cell Mol Life Sci, v. 68, n. 15, p. 2499-512, Aug 2011.

LIN, L.; FARACO, J.; LI, R.; KADOTANI, H.; ROGERS, W.; LIN, X.; QIU, X.; DE JONG, P. J.; NISHINO, S.; MIGNOT, E. The sleep disorder canine narcolepsy is caused by a mutation in the hypocretin (orexin) receptor 2 gene. Cell, v. 98, n. 3, p. 365-76, Aug 61999.

LU, J.; SHERMAN, D.; DEVOR, M.; SAPER, C. B. A putative flip-flop switch for control of REM sleep. Nature, v. 441, n. 7093, p. 589-594, 2006. 
LUPPI, P. H.; CLÉMENT, O.; GARCIA, V. S.; BRISCHOUX, F.; FORT, P. New aspects in the pathophysiology of rapid eye movement sleep behavior disorder: the potential role of glutamate, gamma-aminobutyric acid, and glycine. Sleep Med, v. 14, n. 8, p. 714-8, Aug 2013.

MAQUET, P. The role of sleep in learning and memory. Science, v. 294, n. 5544, p. 1048-52, Nov 22001.

MARKOV, D.; GOLDMAN, M. Normal Sleep and Circadian Rhythms: Neurobiologic Mechanisms Underlying Sleep and Wakefulness. Psychiatric Clinics of North America, v. 29, n. 4, p. 841-853, 2006.

MCCARTER, S. J.; ST LOUIS, E. K.; BOEVE, B. F. REM sleep behavior disorder and REM sleep without atonia as an early manifestation of degenerative neurological disease. Curr Neurol Neurosci Rep, v. 12, n. 2, p. 182-92, Apr 2012.

MCGRANE, I. R.; LEUNG, J. G.; ST LOUIS, E. K.; BOEVE, B. F. Melatonin therapy for REM sleep behavior disorder: a critical review of evidence. Sleep Med, v. 16, n. 1, p. 19-26, Jan 2015.

MOSZCZYNSKI, A.; MURRAY, B. J. Neurobiological aspects of sleep physiology. Neurol Clin, v. 30, n. 4, p. 963-85, Nov 2012.

NISHINO, S.; MIGNOT, E. Pharmacological aspects of human and canine narcolepsy. Prog Neurobiol, v. 52, n. 1, p. 27-78, May 1997.

NISHINO, S.; REID, M. S.; DEMENT, W. C.; MIGNOT, E. Neuropharmacology and neurochemistry of canine narcolepsy. Sleep, v. 17, n. 8 Suppl, p. S84-92, Dec 1994.

PAULETTO, G.; BELGRADO, E.; MARINIG, R.; BERGONZI, P. Sleep disorders and extrapyramidal diseases: an historical review. Sleep Med, v. 5, n. 2, p. 163-7, Mar 2004.

PINTO JR., L. R.; SILVA, R. S. Polissonografia normal e nos principais distúrbios de sono. Medicina e Biologia do sono. TUFIK, S.: Manole Ltda: 161-80 p. 2008.

POPAT, S.; WINSLADE, W. While You Were Sleepwalking: Science and Neurobiology of Sleep Disorders \& the Enigma of Legal Responsibility of Violence During Parasomnia. Neuroethics, v. 8, n. 2, p. 203-214, 2015.

PORKKA-HEISKANEN, T.; STRECKER, R. E.; THAKKAR, M.; BJORKUM, A. A.; GREENE, R. W.; MCCARLEY, R. W. Adenosine: a mediator of the sleep-inducing effects of prolonged wakefulness. Science, v. 276, n. 5316, p. 1265-8, May 231997.

POSTNOVA, S.; VOIGT, K.; BRAUN, H. A. A mathematical model of homeostatic regulation of sleep-wake cycles by hypocretin/orexin. J Biol Rhythms, v. 24, n. 6, p. 523-35, Dec 2009.

PRIMHAK, R.; KINGSHOTT, R. Sleep physiology and sleep-disordered breathing: the essentials. Arch Dis Child, v. 97, n. 1, p. 54-8, Jan 2012.

REITER, R. J.; ROSALES-CORRAL, S.; COTO-MONTES, A.; BOGA, J. A.; TAN, D. X.; DAVIS, J. M.; KONTUREK, P. C.; BRZOZOWSKI, T. The photoperiod, circadian ENCICLOPÉDIA BIOSFERA, Centro Científico Conhecer - Goiânia, v.11 n.22; p.1027 
regulation and chronodisruption: the requisite interplay between the suprachiasmatic nuclei and the pineal and gut melatonin. J Physiol Pharmacol, v. 62, n. 3, p. 269-74, Jun 2011.

RIPLEY, B.; FUJIKI, N.; OKURA, M.; MIGNOT, E.; NISHINO, S. Hypocretin levels in sporadic and familial cases of canine narcolepsy. Neurobiol Dis, v. 8, n. 3, p. 52534, Jun 2001.

SAKURAI, T. Orexin deficiency and narcolepsy. Current Opinion in Neurobiology, v. 23, n. 5, p. 760-766, 2013.

SANTOS, L. C.; CASTRO, N. J.; RUBACK, O. R.; TRIGO, T. J. B.; ROCHA, P. M. B. Transtornos do ciclo sono-vigília/circadiano - uma revisão de literatura. Braz $\mathbf{J}$ of Surg Clin Res, v. 7, n. 2, p. 38-43, 2014.

SAPER, C. B.; CHOU, T. C.; SCAMMELL, T. E. The sleep switch: hypothalamic control of sleep and wakefulness. Trends Neurosci, v. 24, n. 12, p. 726-31, Dec 2001.

SCHENCK, C. H.; HURWITZ, T. D.; MAHOWALD, M. W. Symposium: Normal and abnormal REM sleep regulation: REM sleep behaviour disorder: an update on a series of 96 patients and a review of the world literature. J Sleep Res, v. 2, n. 4, p. 224-231, Dec 1993. ISSN 0962-1105.

SCHENCK, C. H.; MAHOWALD, M. W. REM sleep behavior disorder: clinical, developmental, and neuroscience perspectives 16 years after its formal identification in SLEEP. Sleep, v. 25, n. 2, p. 120-38, Mar 2002.

SCHUBERT, T. A.; CHIDESTER, R. M.; CHRISMAN, C. L. Clinical characteristics, management and long-term outcome of suspected rapid eye movement sleep behaviour disorder in 14 dogs. J Small Anim Pract, v. 52, n. 2, p. 93-100, Feb 2011.

ST LOUIS, E. K. Key sleep neurologic disorders: Narcolepsy, restless legs syndrome/Willis-Ekbom disease, and REM sleep behavior disorder. Neurol Clin Pract, v. 4, n. 1, p. 16-25, Feb 2014.

STEININGER, T. L.; GONG, H.; MCGINTY, D.; SZYMUSIAK R. Subregional organization of preoptic area/anterior hypothalamic projections to arousal-related monoaminergic cell groups. J Comp Neurol, v. 429, n. 4, p. 638-53, Jan 2001.

BJORNESS T. E.; GREENE, R. W. Adenosine and sleep. Curr Neuropharmacol, v. 7, n. 3, p. 238-45, 2009.

THORPY, M. J.; KRIEGER, A. C. Delayed diagnosis of narcolepsy: characterization and impact. Sleep Med, v. 15, n. 5, p. 502-7, May 2014.

TONOKURA, M.; FUJITA, K.; NISHINO, S. Review of pathophysiology and clinical management of narcolepsy in dogs. Vet Rec, v. 161, n. 11, p. 375-80, Sep 2007.

TONONI, G.; CIRELLI, C. Sleep and the price of plasticity: from synaptic and cellular homeostasis to memory consolidation and integration. Neuron, v. 81, n. 1, p. 12-34, Jan 2014. 
TOTH, L. A.; BHARGAVA, P. Animal models of sleep disorders. Comparative Medicine, v. 63, n. 2, p. 91-104, 2013.

TROTTI, L. M. REM sleep behaviour disorder in older individuals: epidemiology, pathophysiology and management. Drugs Aging, v. 27, n. 6, p. 457-70, Jun 2010.

YEO, S. S.; CHANG, P. H.; JANG, S. H. The ascending reticular activating system from pontine reticular formation to the thalamus in the human brain. Front Hum Neurosci, v. 7, p. 416, 2013. 